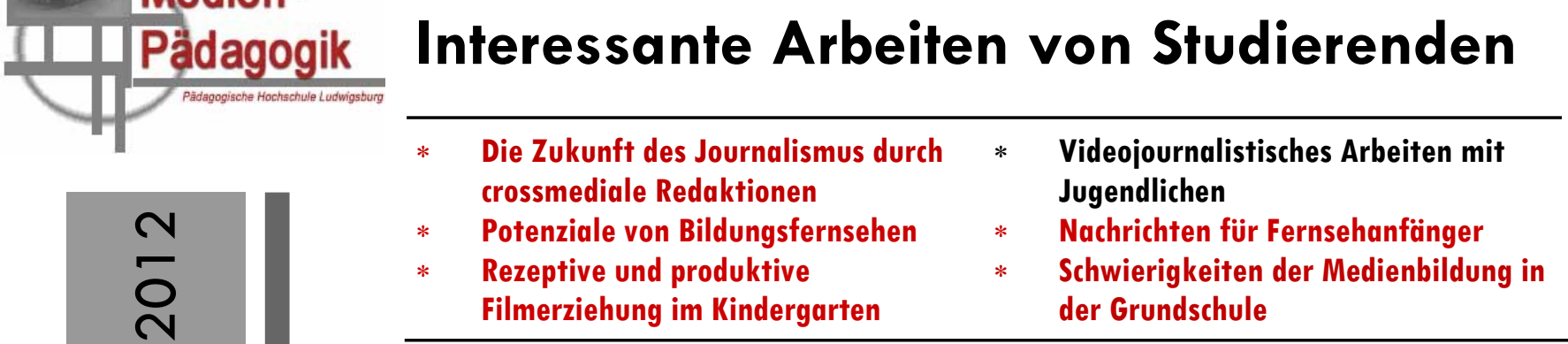

Der folgende Beitrag fasst eine Bachelorarbeit zusammen, die 2012 im Studiengang Kultur- und Medienbildung an der PH Ludwigsburg erstellt wurde (Betreuung: Prof. Dr. Horst Niesyto und Dr. Björn Maurer).

Die Redaktion

Videojournalistisches Arbeiten mit Jugendlichen aus bildungsbenachteiligenden Kontexten. Entwicklung eines medienpädagogischen Konzepts für die schulische und außerschulische Bildungspraxis

\section{NINA WEISENHEIMER}

Während meiner Bachelorarbeit im Studiengang Kultur- und Medienbildung an der Pädagogischen Hochschule Ludwigsburg habe ich mich eingehend mit dem Thema „Videojournalismus für Jugendliche aus bildungsbenachteiligenden Kontexten" auseinandergesetzt. Der folgende Artikel bezieht sich auf die Ausarbeitung des Themas und stellt einen Überblick über meine Ergebnisse und Ableitungen dar. Alle Ergebnisse fließen anschließend in eine konkrete Ausarbeitung eines medienpädagogischen Konzeptes für die schulische und außerschulische Bildungspraxis ein.

Der Erwerb folgender Kompetenzen, die für den Lebensweg der Zielgruppe wichtig sind, stehen im Mittelpunkt der Konzeption:

Medienkompetenz: Technikkompetenzen in den Bereichen Kamera, Ton und Videoschnitt, medienkritische Aspekte, mediengestalterische Fähigkeiten.

Journalistische Skills: Schreiben fürs Fernsehen, Recherche, Interviews führen, Beiträge gestalten.

Soft Skills und Persönlichkeitsbildung: Gruppenarbeit, Allgemeinwissen, Organisation, Konzentration, Kommunikation, schriftlicher und verbaler Ausdruck.

Ein weiterer - sehr wichtiger - Aspekt, den das journalistische Arbeiten innehat und der für Jugendliche aus bildungsbenachteiligenden Kontexten eine große Chance bietet, ist die aktive Mitsprache, das Sprachrohr, wel- ches die videojournalistische Arbeit bietet: Die Jugendlichen erhalten die Möglichkeit, einer Öffentlichkeit ihre Ansichten und Meinungen mitzuteilen - und erarbeiten sich ein Stück Mitsprache und die Möglichkeit zur Teilhabe in einer partizipativen Demokratie.

„Soziale Ungleichheit im weiteren Sinne liegt überall dort vor, wo die Möglichkeit des Zuganges zu allgemein verfügbaren und erstrebenswerten sozialen Gütern und/oder sozialen Positionen, die mit ungleichen Macht- und/oder Interaktionsmöglichkeiten ausgestattet sind, dauerhafte Einschränkungen erfahren und dadurch die Lebenschancen der betroffenen Individuen, Gruppen oder Gesellschaften beeinträchtigt bzw. begünstigt werden“ (Kreckel 2004, S. 17).

Benachteiligung liegt vor, ,wenn bestimmten sozialen Gruppen der Zugang zu gesellschaftlich relevanten Werten und Ressourcen, zum Beispiel höheres Einkommen, soziale Sicherheit, aber auch Bildung, durch Schichtgrenzen und/oder Diskriminierung verwehrt bleibt oder erschwert ist" (Niesyto 2004, S. 124).

Hier zeigt sich also, dass Bildungsbenachteiligung und soziale Ungleichheit eine Spirale bilden, die unendlich fortgesetzt werden kann: Bildungsbenachteiligung führt $\mathrm{zu}$ sozialer Benachteiligung, soziale Benachteiligung wiederum zu Bildungsbenachteiligung.

Diese Erkenntnisse fasst Duncker sehr treffend und prägnant zusammen, indem er schreibt, „[...] dass sich im Bereich der Hauptschule zahlreiche soziale Probleme unserer Gesellschaft in dichter Form widerspiegeln, dass wir in den Hauptschulbildungsgängen deshalb überdurchschnittlich viele Folgen schwieriger Verhältnisse des Aufwachsens beobachten können und dass wir es bei Hauptschülerinnen und -schülern eher mit Verlierern als Gewinnern in Schule und Gesellschaft zu tun haben" (Duncker 2003, S. 3).

Die 16. Shell-Jugendstudie 2010 erstellt basierend auf den bevorzugten Freizeitaktivitäten von Jugendlichen vier unterschiedliche Typen: die kreative Freizeitelite, die engagierten Jugendlichen, die medienfixier- 
ten und die geselligen Jugendlichen, wobei sich die Jugendlichen anteilig fast genau auf die vier Gruppen aufteilen (Anteile jeweils zwischen 23 - $28 \%$ ) (vgl. Leven u. a. 2010, S. 98f.). Unter den Jugendlichen, die der Unterschicht zugeordnet werden können, liegt der Anteil der „Medienfixierten“ mit fast 47 \% höher als der Durchschnitt. Die Hauptfreizeitaktivitäten der Medienfixierten sind Fernsehen (84\%), im Internet surfen $(77 \%)$, Musik hören (79 \%), DVD (47\%) und Rumhängen $(33 \%)$. Eher abgeneigt ist diese Gruppe gegenüber Sport, Büchern, Unternehmungen mit der Familie und Kreativem (vgl. Leven u.a. 2010, S. 98f.). Weitere spannende Werte zur Mediennutzung von Jugendlichen aus bildungsbenachteiligenden Kontexten finden sich auch in der aktuellen JIMbzw. FIM-Studie, auf welche an dieser Stelle nicht genauer eingegangen wird.

Daraus lässt sich für die medienpädagogische Arbeit ableiten, dass die Jugendlichen sehr medienaffin sind und sich mit den Medien (zumindest im rezeptiven Bereich) gut auskennen.

Da Jugendliche aus bildungsbenachteiligenden Kontexten potenziell von mehreren benachteiligenden Faktoren betroffen sind, gilt es hier gezielt gegenzusteuern. Die Jugendlichen, die oft in einer bildungsarmen und hindernisreichen Lernumwelt aufwachsen, müssen animiert werden. Es ist wichtig, ihnen neue bzw. andere soziale Handlungsfelder und Räume aufzuzeigen, deren Zugang nicht durch Anmeldeverfahren oder gar Vorwissen versperrt wird (Niederschwelligkeit und Erreichbarkeit der Angebote). Da der klassische Schulunterricht oftmals mit einem hohen $\mathrm{Maß}$ an Frustration verknüpft ist, eignen sich Lehrmethoden, die an ihrer (medialen) Lebenswelt anknüpfen und frei sind vom klassischen schulischen Leistungsdruck. Das Interesse und die Nutzungsfrequenz an Medien im Allgemeinen ist sehr hoch, wie durch die JIMStudie und die Shell-Jugendstudie gezeigt werden konnte. Götz und Durner sehen die Chance der Medienbildung - gerade für bildungsferne Jugendliche - in der Lebensweltnähe (vgl. Götz/ Durner 2007, S. 58).

Für das videojournalistische Arbeiten mit Jugendlichen aus bildungsbenachteiligenden Kontexten eignen sich von daher Themen an, die sie direkt betreffen, bei denen sie aufgrund ihrer persönlichen Erfahrungen mitsprechen können, ohne auf „Wissen aus dem Schulbuch“ zurückgreifen zu müssen. Über diesen Zugang lassen sich auch abstraktere Themen weiter ausbauen und erarbeiten.

Da Jugendliche aus bildungsbenachteiligenden Kontexten tendenziell weniger Interesse an Textmedien haben, eignen sich Arbeits- weisen, die stärker auf kreativ-gestalterischen, sprachlichen oder gar körperlichen Ausdruck ausgerichtet sind. Für die konkrete Arbeit bedeutet das, dass auf lange Skripte, die durchgelesen werden müssen oder Frontalunterricht, der wenig Raum für kreative Mitarbeit lässt, als Hauptform verzichtet werden sollte.

Niesyto stellte in seinem Vortrag auf der Fachtagung „Anschluss oder Ausschluss? Medien und Benachteiligtenförderung“" am 17.10.2002 konkrete Kriterien für die gelungene Medienbildung mit Jugendlichen aus bildungsbenachteiligenden Kontexten vor (vgl. Niesyto 2002, S. 11ff.), welche die von mir erarbeiteten Kriterien erweitern und vervollständigen:

\section{- Dezentrale Zugangsformen}

- Erfahrungs- und Lebensweltorientierung

- Präsentative Ausdrucksformen stärker integrieren: „Meine These ist, dass ein integriertes Konzept einer umfassenden 'Alphabetisierung' notwendig ist, das diskursive und präsentative Ausdrucksund Kommunikationsformen in eine Balance bringt, das ein Navigieren mit diskursiven und präsentativen Codes ermöglicht. Sehr viele Jugendliche haben Stärken im präsentativen Bereich - wir sollten endlich Konzepte stärker entwickeln, die an diesen Fähigkeiten und vorhandenen Kompetenzen anknüpfen und sie durch fachliche Inputs konsequent fördern.“ (Niesyto 2002, S. 12)

- Ästhetisch-kulturelle und arbeitsweltbezogene Kompetenzen miteinander verbinden: Die Kompetenzen, die Jugendliche bei der Gestaltung mit Medien erwerben, sind wichtig für ihre spätere berufliche Laufbahn. Niesyto nennt hier vor allem sozial-kommunikative, ästhetische, technische und methodische Kompetenzen, deren Erwerb bei der Konzeption stärker ins Gewicht fallen sollte (vgl. Niesyto 2006, S. 45).

- Spielerische und non-lineare Arbeitsweisen fördern

- Präsentation und Kommunikation lernen

- Die Mittelschicht-Lastigkeit in der (Medien-)Pädagogik überwinden

Diese Auffassung findet sich auch bei Lauffer, der gerade in der Möglichkeit, abseits von „auf Sprache gestützten Konzepten“ in einer „Kombination unterschiedlicher kreativer Methoden, z.B. von Theaterarbeit mit journalistischen Techniken oder mit Kunst sowie musischer Bildung“ (Lauffer 2006, S. 38) für 
Jugendliche bildungsferner Schichten großes Potenzial sieht.

Diemand nennt als Eigenschaften, die zum zunehmenden Einsatz von Fernsehjournalisten geführt haben, „die immense Kostenersparnis, welche sowohl für non-profit- wie for-profit-Akteure von Bedeutung ist" (Diemand u. a. 2007, S. 9), sowie die kleinen handlichen DV-Kameras, die es dem Videojournalisten ermöglichen, eine neue Nähe zum Geschehen aufzubauen und so eine neue Ästhetik schaffen (vgl. Zalbertus/Rosenblum 2004, S. 42).

Ein großes Kamerateam kann - im Gegensatz dazu - oft einschüchternd für den Protagonisten wirken und ihn hemmen. Dazu ermöglichen Innovationen im Bereich der $\mathrm{Ka}$ meratechnik, dass mit kleinen Kameras für verhältnismäßig wenig Geld Bilder in HD-Qualität erstellt werden können: Mit der neuen Technik kann jeder qualitativ hochwertige Beiträge erstellen, es stellt somit eine wirkliche Alternative zum großen und teuren Profiequipment dar.

Videojournalismus ist - zusammenfassend nicht nur eine neue Arbeitsweise des Journalismus, sondern produziert auch seine eigene Ästhetik und Gestaltungsformen. Die Arbeit des Videojournalisten umfasst alle Schritte von der Konzeption bis zum Schnitt eines Beitrages. So ist es Videojournalisten möglich, schnell zu reagieren und flexibel auf das Thema einzugehen.

\section{Medienpädagogisches Konzept für die schulische und außerschuli- sche Bildungspraxis}

Das Konzept gliedert sich in mehrere Module, die untereinander je nach Bedarf und Zeitfenster kombiniert werden können. Bei der Planung des Gesamtumfangs des Projektes ist zu bedenken, dass die Dauer der Module im Vergleich zu der Zeit, in der der videojournalistische Beitrag konzipiert, gedreht und geschnitten wird, relativ gering ist. Den größten Teil nehmen die Themenfindung, der Dreh, der Schnitt und die Nachbearbeitung in Anspruch. Hier sollte der Anspruch an das fertige Produkt, vor allem im Bezug auf den Umfang, an die zeitlichen Gegebenheiten angepasst werden. Ist zum Beispiel nur ein Nachmittag für den Dreh einkalkuliert, muss genau geplant werden, an wie vielen Plätzen man drehen kann und wie viele Interviewpartner man in der Zeit „schafft“. Auch die Dauer der einzelnen Treffen sollte nicht kürzer als 1,5 Stunden sein. Gerade für die Arbeit mit der Kamera sind größere Zeitfenster wichtig, da der Aufbau der Technik, die tatsachliche Arbeit und eine anschließende Reflexion in einem Zeitfenster unter 1,5 Stunden nur unter Zeitdruck realisierbar sind.

Die Stoffmenge wird nach dem Prinzip der didaktischen Reduktion an die Zielgruppe angepasst. Somit wird in einigen Bereichen (zum Beispiel im Modul Drehtag) mit einfachen Merksätzen und Zusammenfassungen gearbeitet. Ein Beispiel für eine didaktische Reduktion in diesem Konzept ist der Merksatz „Kein Zoom bei eingeschalteter Kamera".

In der ausführlichen Beschreibung des Projektes werden noch ergänzende Hinweise und Anregungen zu theaterpädagogischen Warmups (Ankommen, Kennenlernen) sowie zur Reflexion und dem Abschluss der Moduleinheiten gegeben. Ebenfalls werden ausführliche Hinweise zu rechtlichen Rahmenbedingungen gegeben, die hier aus Platzgründen ausgespart werden.

Das Konzept ist für Jugendliche aller Altersstufen ab ca. Klasse 7 gleichermaßen geeignet. Viele der angesprochenen Themen und Lernziele sind exemplarisch im Bildungsplan der Werkrealschulen für Baden-Württemberg ab Klasse 7 verankert.

Vorab sollte besprochen werden, ob es zu dem Beitrag ein „Making-Of“ geben soll. Das Making-Of zeigt die Jugendlichen bei der Arbeit an dem Beitrag. Das bedeutet, dass die Jugendlichen sich selbst bei der Arbeit filmen, es entsteht eine weitere Dokumentation. Die Jugendlichen, die das Making-Of drehen, sind immer mit der Kamera dabei. Ergänzend dazu können auch Interviews aufgenommen werden oder lustige „Outtakes“ aus der Produktion beigefügt werden. Der Vorteil ist, dass das Making-Of eine weitere Möglichkeit der Reflexion bietet, dazu üben die Jugendlichen konstant mit der Kamera weiter. Das Making-Of $\mathrm{zu}$ drehen und $\mathrm{zu}$ schneiden ist zwar noch einmal sehr viel Arbeit, ist aber eine schöne Erinnerung an die Produktion und letztendlich auch ein Stück Anerkennung und Wertschätzung der Arbeit der Jugendlichen.

Im Folgenden werden die Module des Projektes kurz vorgestellt.

Modul 1 - Kamera

Um einen vermeintlich langweiligen oder textlastigen Einstieg zu verhindern, wird bereits im ersten Modul die Kamera eingeführt. So haben die Teilnehmer schon bei Projektstart die Möglichkeit, zu üben und zu experimentieren. Empfehlenswert ist eine formale Aufgabenstellung. Das bedeutet, die Jugendlichen bekommen ihre Aufgabe im gestalterischen Bereich, nicht im thematischen Bereich: Zum Beispiel sollen sie einen Kamera- 
schwenk aufzeichnen oder - für Fortgeschrittene - eine Plansequenz, erhalten hierzu aber keine verbindliche Aufgabe zum thematischen Inhalt. So haben die Jugendlichen von Anfang an die Möglichkeit, ihre eigenen Themen zu bearbeiten und zu zeigen (vgl. Maurer 2001, S. 5). Wichtig ist auch, das entstandene Material gleich zu begutachten, da eine schnelle Rückmeldung für die Zielgruppe wichtig ist. Hierzu bietet sich die Betrachtung mit einem Beamer an. So entsteht auch gleich die Möglichkeit, anhand des entstandenen Materials typische „Anfängerfehler“ zu thematisieren und mit der Gruppe zusammen nach Lösungen zu suchen. Dieses Herangehen entspricht der handlungsorientierten Pädagogik, die Jugendlichen lernen direkt aus der eigenen Erfahrung.

\section{Modul 2 - Interviewfübrung und das Mikrofon}

Interviews führen ist oftmals nicht so einfach wie angenommen. Was im Fernsehen immer sehr locker und spontan wirkt, beinhaltet eine Menge Vorbereitung und Übung. Vor einem Interview sollte ein Leitfaden erarbeitet werden, der dem Reporter hilft (vgl. Anfang u.a. 2006, S. 49).

Um mit der Gruppe die Interviewtechnik zu erarbeiten, bietet es sich an, Interviews auszuwerten. Viel Material bietet zum Beispiel die Seite der Kinderreporter des ZDFtivi!. Die Interviews können unter folgenden Gesichtspunkten angesehen und ausgewertet werden:

Fragetechnik: Welche Fragen werden gestellt? Warum wurden diese Fragen gestellt? Werden alle Fragen beantwortet? Was hätte man noch fragen können?

Darstellung: Welche Bilder haben wir außerhalb der Interviewsituation gesehen? Warum wurden diese gewählt?

Journalistische Auffassung: Wie verhalten sich die Kinderreporter gegenüber dem Interviewpartner? Wie sprechen sie den Interviewpartner an? Wie begegnen sie ihm?

Allgemeines: War das Interview spannend? Hast Du etwas Neues erfahren/ gelernt? Was hat gestört?

Übertragung: Was hättest Du den Interviewpartner gerne gefragt? Was hättest Du gerne noch gewusst?

Auch bei diesem Modul sollte nach einer kurzen Einführung in die Technik (Anstecken des Mikrofons, Einschalten, Pegel prüfen etc.) am besten durch eigenständiges Probieren gelernt werden. Die Jugendlichen sollen sich gegenseitig zu einem vorher in der Gruppe besprochenen Thema interviewen. Sie bekommen ein Arbeitsblatt, auf dem sie vorab die Fragen eintragen sollen. Dieser „Leitfaden“ hilft den Jugendlichen beim Interview selbst und dient als Gedankenstütze und Orientierung (vgl. Anfang u.a. 2006, S. 49).

Darauf aufbauend kann man bereits unterschiedliche Fragemethoden besprechen.

Das Ziel des Moduls ist erreicht, wenn die Jugendlichen anschließend eine Vorstellung von einem gelungenen Interview haben und sich gegebenenfalls schon ein paar Gedanken über ihre eigenen Interviews gemacht haben.

Modul 3 - Themenfindung

Gerade in einer größeren Gruppe ist eine gemeinsame Themenfindung oftmals problematisch. Da das Thema für die nächsten Wochen oder Monate sehr viel Raum einnehmen wird, ist es wichtig, dass alle Jugendlichen hinter dem Thema stehen.

Eine Methode ist die Arbeit über Mindmaps. Die Jugendlichen erhalten in Kleingruppen ein noch sehr unspezifisches Themenfeld (z.B. meine Freizeit, meine Hobbys, mein Schulalltag) und sammeln dazu Begriffe. Die Begriffe gliedern sie in sinnverwandte Bereiche. Anschließend werden die Mindmaps aufgehängt und zur Diskussion freigegeben. Gibt es thematische Überschneidungen? Gibt es Themen, die für alle interessant sind? Lassen sich Themen kombinieren? Unter Umständen steht als Aufgabenstellung ein bestimmtes Ereignis im Vordergrund, welches dokumentiert werden soll (das Schulfest, die Projektwoche etc.). Auch hier wird zuerst überlegt, welche Hintergrundthemen interessant sind. So erschließt sich relativ schnell ein Gesamtbild. Ist das Thema gefunden ist der nächste Schritt die Recherche.

Modul 4 - Journalistisches Arbeiten: Recherche und Co.

Was bedeutet eigentlich Recherchieren? Und wie wird es gemacht? In diesem Modul lernen die Jugendlichen, wie man - hauptsächlich im Internet - an Informationen gelangt.

Bei aktuellen Themen ist die Recherche in der Zeitung oder in Magazinen hilfreich. Diese Informationen können wiederum mit Informationen aus dem Internet ergänzt wer- den (vgl. Anfang u.a. 2006, S. 44f.). Auch sollte bei der Recherche nicht die Recherche in der Bibliothek vergessen werden. Da die teilnehmenden Jugendlichen tendenziell eher selten lesen, kann die Bibliothek eventuell neu entdeckt werden und ihnen neue Möglichkeiten und Lernräume aufzeigen.

Auch die Recherche direkt vor Ort bietet viele Möglichkeiten. Die Jugendlichen lernen gleich den Drehort kennen und haben so die Möglichkeiten, sich bereits vorab vorzubereiten. Dazu können im direkten Gespräch mit den Interviewpartnern und anderen beteiligten Personen, Schwerpunkte der Produktion 
gesetzt werden oder die Jugendlichen werden auf Themenbereiche oder Aspekte aufmerksam, die sie noch nicht beachtet hatten (vgl. Anfang u.a. 2006, S. 46). So wird auch bereits der Kontakt zu Experten beziehungsweise Interviewpartnern hergestellt. Die Jugendlichen sollten, bevor sie mit den Experten in Kontakt treten, bereits mit dem Thema vertraut sein und einen Überblick über die Fragen an den Experten haben.

Der Lerneffekt dieser Übung: Quellen auswerten, Wissen zusammenfassen und kürzen, Quellen vergleichen, sich sicher und zielgerichtet im Internet bewegen.

Modul 5 - Drehplan, Script, Storyboard

Bei Filmproduktionen ist eine gute Vorbereitung des Drehs das A und O. Anders wie beim Spielfilm lässt sich beim dokumentarischen Arbeiten aber nicht alles planen. Die Kamera muss flexibel auf das Geschehen reagieren. Dennoch ist es sinnvoll, vor dem Drehtag eine Checkliste zu erstellen, welche Aufnahmen benötigt werden.

Auch die 5-Shot-Technik kann genutzt werden, wenn die Kameraleute am Drehort schnell auf ein Geschehen reagieren sollen. Als Handlungsanweisung sollten alle fünf Einstellungen nach der 5-Shot-Technik aufgezeichnet werden. Hat man diese Einstellungen sicher im Kasten, entstehen weniger Anschlussfehler beim Schnitt. Die Dokumentation folgt einer Dramaturgie. Einleitend sollten Gegebenheiten wie Ort, Personen und das Thema vorgestellt werden. Hier soll das Interesse des Zuschauers geweckt werden. Anschließend werden Argumente und Blickwinkel auf das Thema vorgestellt. Am besten bauen die Argumente aufeinander auf oder beziehen sich aufeinander (vgl. Anfang u.a. 2006, S. 47). Das Ende stellt den Höhepunkt dar: "Hier muss die zentrale Aussage des Filmes formuliert und der dramaturgische Höhepunkt erreicht warden" (Anfang u.a. 2006, S. 49). Am Ende soll möglichst eine Aussage stehen, die für alle Zuschauer einen Informationsgewinn darstellt (vgl. Anfang u.a. 2006, S. 47). Eine weitere Möglichkeit ist das experimentelle Arbeiten. Hier wird stark assoziativ und mit einer experimentellen, ungewöhnlichen Ästhetik gearbeitet. Experimentelle Dokumentarfilme sind stark assoziativ und wollen beim Zuschauer Emotionen wecken. Sie handeln oft von Gefühlen, Stimmungen oder eher abstrakteren Themen. Ein Merkmal des experimentellen Arbeitens ist eine aufwendigere Postproduktion im Sound- und Bildbereich (Arbeit mit Farboder Toneffekten) (vgl. Anfang u.a. 2006, S. 42). Die Stärke des experimentellen Arbeitens für die Zielgruppe ist, dass ihrem Selbstausdruck fast keine Grenzen gesetzt sind. Diese Vorgehensweise baut mehr auf einen spielerisch-kreativen Zugang auf. Ziel dieses Moduls ist es, zusammen mit den Jugendlichen eine Checkliste für den Drehtag zu erarbeiten, die am Drehtag (natürlich nicht ausschließlich) abgearbeitet werden kann und somit Orientierung bietet.

Modul 6 - Unterwegs mit der Kamera - Der Drehtag

Die Vorbereitungen für den Drehtag beginnen, je nachdem, wo gedreht wird, mit dem Einholen der Drehgenehmigungen und der Terminabsprache mit den Interviewpartnern. Hier sollte man rechtzeitig alle Formalitäten klären, damit am Drehtag alles reibungslos läuft (vgl. Anfang u.a. 2006, S. 52). Die Jugendlichen sind dazu angehalten, alle organisatorischen Aufgaben in Eigenverantwortung zu erledigen, dennoch sollte der Projektleiter beratend und unterstützend zur Seite stehen und alle Aufgaben im Kopf haben. Auch kann die organisatorische Arbeit unter Umständen sehr frustrierend sein: Nicht jeder hat Zeit und Lust an einem Interview mitzuwirken. Am Drehtag sollte darauf geachtet werden, dass genügend Füllbilder, sowie „Raumatmo“ aufgezeichnet werden. Auch sollte die Checkliste (siehe Modul 4 - Drehplan, Script, Storyboard) mitgenommen und eingehalten werden, ebenso die Leitfäden für die Interviews. Vor dem Dreh gilt es, die Technik zu überprüfen: Ist alles komplett? Ist der Akku geladen? Ist die Speicherkarte leer (oder haben wir genügend leere Bänder)? Es gibt nichts was mehr ärgert, als ein leerer $\mathrm{Ak}$ ku beim Dreh (vgl. Anfang u.a. 2006, S. 51).

\section{Modul 7 - Und jetzt? Material sichten und schneiden}

Normalerweise hat man viel mehr Material, als man im Beitrag verwenden kann. Dieses gilt es nun zu selektieren und zu bewerten. Je nach Zeitrahmen kann der Projektleiter vorab einen Grobschnitt vornehmen und der Gruppe ein bereits selektiertes Material zum Schnitt zur Verfügung stellen.

Bei der musikalischen Untermalung muss an das Urheberrecht gedacht werden. Um Problemen mit dem Urheberrecht aus dem Weg zu gehen, eignen sich freie Musik oder Musik, die unter einer Creative-Commons-Lizenz verwendet werden kann. Hierzu gibt es im Internet einige Portale, die freie Musik (und auch Geräusche) anbieten. Selbstverständlich kann die Gruppe auch selbst die Filmmusik komponieren oder einsingen. So haben die Jugendlichen erneut die Chance ihre Talente zu zeigen.

Modul 8 - Wir brauchen mebr Info: Der Sprechertext

Beim Sprecher unterscheiden wir zwischen einem On- und einem Off-Sprecher. Der Off 
-Sprecher erscheint nur auf der Ton-ebene und erscheint nicht im Bild. Der On-Sprecher wird auf der Bildebene gezeigt, meist am Ort des Geschehens. Der Sprechertext kann dazu verwendet werden, den Beitrag mit Informationen zu ergänzen, die auf der Bildebene nicht gezeigt werden. Hier empfiehlt es sich, sich an die gängigen Regeln des „Schreiben fürs Hören" zu halten.

Schneider und Raue empfehlen für Attraktivität und Verständlichkeit von Texten zum Hören und Lesen ,die konkretesten, die schlichtesten möglichen Wörter in schlanken, transparenten Sätzen“ (Schneider/ Raue 2012, S. 42). Das bedeutet:

- Keine langen Schachtelsätze, um die Verständlichkeit zu erhöhen.

- Der Text sollte so nah wie möglich an der natürlichen Sprache des Sprechers liegen. Fremdwörter und Fachbegriffe sparsam einsetzen, wenn nötig erklären.

- Auf Passivkonstruktionen und Subjektivierungen wenn möglich verzichten.

- Informationen „portionieren“ und nicht auf einmal in einen Satz packen, um den Zuschauer nicht zu überfordern.

Zusätzlich sollte noch auf ein angemessenes Tempo des Sprechers geachtet werden. Die Aufnahme des Off-Sprechertextes sollte, wenn kein Aufnahmeraum zur Verfügung steht, in einem möglichst kleinen Raum (weniger Hall) und vor allem ruhigen Raum erfolgen. Die Jugendlichen sollten vor dem Mikrofon (oder dem mp3-Aufnahmegerät) stehen. Sollten sie von einem Blatt ablesen, sollten sie das Blatt vor sich ablegen und es nicht in der Hand halten, meist hört man sonst ein Rascheln im Hintergrund. Die Sätze können mehrfach nacheinander eingesprochen werden, bis man mit der Aufnahme zufrieden ist. Fehlversuche können anschließend einfach geschnitten werden. Steht keine Software für die Audioaufnahme zur Verfügung, empfiehlt sich z.B. die OpenSource-Software "Audacity" für Audioaufnahmen und Audioediting. Allerdings kann der Sprecher auch im Bild erscheinen und moderieren. Dann bietet es sich an, die Moderation am "Ort des Geschehens" aufzuzeichnen.

Modul 9 - Unser Film: jetzt auf der Leinwand, online oder gebrannt?

In der Produktion des Beitrages - oder gar der Sendung - steckt viel Arbeit, Zeit und Mühe. Dies sollte anerkannt werden. Um die Produktion im kleinen Rahmen öffentlich zu machen, bieten sich folgende Wege an:

- (öffentliche) Filmvorführung mit anschlie-
Bender Gesprächsrunde im Jugendhaus, in der Aula etc.

- Veröffentlichung auf einem Video-Portal wie z.B. YouTube oder vimeo.

- DVD: Jeder Jugendliche erhält für zu Hause eine DVD mit dem Beitrag.

\section{Literatur}

Anfang, Günther/ Bloech, Michael/ Hültner, Robert (2006). Vom Plot zur Premiere. Gestaltung und Technik für Videogruppen. 2. Auflage. München: kopaed.

Becker, Rolf/ Lauterbach, Wolfgang (2010). „Bildung als Privileg - Ursachen, Mechanismen, Prozesse und Wirkungen“. In: Becker, Rolf / Lauterbach, Wolfgang (Hrsg.): Bildung als Privileg. Erklärungen und Befunde zu den Ursachen der Bildungsungleichheit. Wiesbaden: VS Verlag, S. 11-49.

Diemand, Vanessa/ Mangold, Michael/ Weibel, Peter (Hrsg.) (2007). Weblogs, Podcasting und Videojournalismus. Hannover: Heise Zeitschriftenverlag GmbH \& Co. KG.

Duncker, Ludwig (Hrsg.) (2003). Konzepte für die Hauptschule: Ein Bildungsgang zwischen Konstruktion und Kritik. Bad Heilbrunn: Verlag Julius Klinkhardt.

Götz, Maya/ Durner, Alexandra (Hrsg.) (2007). Journalismus mit Jugendlichen für Jugendliche. Politische Medienbildung in der Schule. Internationales Zentralinstitut für Jugend- und Bildungsfernsehen. München: kopaed.

Kreckel, Reinhard (2004). Politische Soziologie der sozialen Ungleichheit. Frankfurt/ Main: Campus Verlag.

Lauffer, Jürgen (2006). „Aktuelle Rahmenbedingungen medienpädagogischer Arbeit". In: Lauffer, Jürgen/ Röllecke, Renate (Hrsg.): Methoden und Konzepte medienpädagogischer Projekte - Dieter Baacke Preis Handbuch 1. Bielefeld: GMK Gesellschaft für Medienpädagogik und Kommunikationskultur, S. 37-41.

Leven, Ingo/ Quenzel, Gudrun/Hurrelmann, Klaus (2010). Familie, Schule, Freizeit: Kontinuität im Wandel. In: Shell Deutschland Holding (Hrsg.) 16. Shell Jugendstudie. Jugend 2010. Frankfurt/Main: Shell Deutschland Holding $\mathrm{GmbH}$ und Fischer Taschenbuch Verlag, S. 53-128.

Löw, Martina (2003). Einführung in die Soziologie der Bildung und Erziehung. Opladen: Leske + Budrich.

Maurer, Björn (2001). Was willst Du mal werden? Videoprojekt in der Hauptschule. In: Fachstelle Medien der Diözese RottenburgStuttgart. Medienpädagogische Praxismodelle 
Heft 1. Stuttgart, S. 3-12. URL: http:// www.drs.de/index.php?id=1737 (besucht am 26.04.2012).

Niesyto, Horst (2002). Digitale Spaltung digitale Chancen. Medienbildung mit Jugendlichen aus benachteiligenden Verhältnissen. Vortragsmitschrift Fachtagung Anschluss oder Ausschluss? Medien und Benachteiligtenförderung am 17.10.2002. URL: http: // www.mediaculture-online.de/fileadmin/ bibliothek/niesyto_medienbildung/ niesyto_medienbildung.html (besucht am 22.04.2012).

Niesyto, Horst (2004). Medienbildung mit Jugendlichen in Hauptschulmilieus. In: Otto, Hans-Uwe/ Kutscher, Nadja (Hrsg.): Informelle Bildung Online. Perspektiven für Bildung, Jugendarbeit und Medienpädagogik. Weinheim und München: Juventa Verlag, S. 122-136.

Niesyto, Horst (2006). Projektarbeit mit Kindern und Jugendlichen aus bildungsmäßig benachteiligenden Milieus. In: Lauffer, Jürgen/ Röllecke, Renate (Hrsg.): Methoden und Konzepte medienpädagogischer Projekte - Dieter Baacke Preis Handbuch 1. Bielefeld: GMK Gesellschaft für Medienpädagogik und Kommunikationskultur, S. 42-50.

Renner, Karl Nikolaus (2007). Fernsehjournalismus. Entwurf einer Theorie des kommunikativen Handelns. Konstanz: UVK Verlagsgesellschaft.

Schneider, Wolf/ Raue, Paul-Josef (2012). Das neue Handbuch des Journalismus und des Online-Journalismus. Bonn: Bundeszentrale für politische Bildung.

Statistisches Bundesamt und Wissenschaftszentrum Berlin für Sozialforschung (2011). Datenreport 2011. Ein Sozialbericht für die Bundesrepublik Deutschland. Bonn: Bundeszentrale für politische Bildung.

Zalbertus, Andre/ Rosenblum, Michael (2004). Video Journalism. The Digital Revolution. Berlin: uni-edition $\mathrm{GmbH}$. 